\title{
EDITORIAL
}

\section{Evaluation of psychotherapy}

Of all forms of treatment, in all of medicine, psychotherapy is perhaps the hardest to comprehend. In its most extensive meaning, its history is as old, and its scope as wide, as medicine itself, and it is practised with more or less awareness by all doctors. The concept is difficult to elucidate at this level; and though most would regard its importance as self-evident, it is not susceptible to rigorous evaluation except perhaps in those limited situations where automation precludes the inevitability of a doctorpatient relationship. In a stricter sense, psychotherapy comprises an assemblage of methods and skills, important in helping patients whose illnesses have evident psychological causes and manifestations, which can be designatedrather unsatisfactorily-as the directive and supportive psychotherapies. These methods, closely interwoven into general psychiatric practice, lay emphasis on the painstaking application of such imponderables as friendly interest, concern, sensitivity and responsiveness to the patient's feelings, empathy, informed judgment, reflective advice, practical suggestions, and manifest respect for the patient's individuality. Their value in principle seems clear enough. Although these and similar variables can be studied, and to some extent measured and manipulated in clinical research, their absolute withdrawal for experimental purposes would generally be unethical. In its third, and most restricted usage, psychotherapy denotes highly developed and skilled psychological techniques for treating certain patients with specified disabilities. Behavioural therapies can be included under this heading. So are formal, analytic or 'dynamic' psychotherapies-the adjectives are here used synonymously - which are practised mainly by those with a special training, often involving a personal psychoanalysis. The techniques of dynamic psychotherapy, like those of behaviour therapy, are based on theoretical postulates and directed towards helping defined groups of patients; they are specific forms of treatment and, therefore, their effectiveness should be demonstrable.

These three domains of psychotherapy, each distinguished from the others by the context in which it is practised and by its manifest purpose, share much common ground. Transactions between doctors and patients-like all but the most casual of relationships-have always been affected, in conspicuous or hidden ways, by those beliefs and attitudes which are fashionable in a society. And the contemporary climate of opinion in most Western societies has been deeply influenced by the emergence of psychoanalysis as a model or metaphor for understanding human experience, relationships, development, and behaviour.

Partly as a consequence of this, two claims are made for the relevance of modern psychodynamic concepts to medicine. One is that they have a very wide significance in the management of patients, and therefore a large value in medical education. According to this idea, the practice of medicine in general, and psychiatry in particular, makes demands on certain important qualities of the practitioners. These qualities, it is suggested, can be communicated, evoked or facilitated during training, specifically by exposure to specialized teaching in psychodynamics, and by this means alone. The other claim is that dynamic psychotherapy is an effective and important specific treatment for certain patients. Both claims are so far-reaching as to call for close and dispassionate study. The need for careful examination of these intricate matters becomes even more clamant when seen in the light of developments in undergraduate medical education, postgraduate education in psychiatry, and in the planning of psychiatric services.

In evaluating dynamic psychotherapy, five questions are at issue. Do patients chosen for this type of treatment fare better than they would if treated differently? What components of treatment determine its effectiveness? What proportion of patients with defined forms of 
disability are suitable for one or other kind of dynamic psychotherapy, and how may they be identified? For how many suitable patients is this treatment available? Finally, when dynamic psychotherapy is available to a community, are matters of suitability and responsiveness to be regarded as stable qualities, or are they subject to subcultural influences such as the caprices of fashion and other psychosocial variables? The last four questions are cogent only if an affirmative answer to the first is demonstrated or assumed.

Many formidable difficulties are involved in investigating the effectiveness of dynamic psychotherapy. The most outstanding are those of specifying, with sufficient exactitude, the components of treatment, the disabilities for which they are employed, and the objectives to be attained. Dynamic psychotherapy is the name given to a large class of treatments, in which the most obvious feature is that the patient has more and longer interviews than is the rule with treatments more generally available in the National Health Service. Less conspicuously, a special kind of relationship develops between the therapist and the patient (or group of patients), in which complex verbal exchanges and emotional interactions take place; and treatment is conducted in accordance with certain postulates deriving ultimately from psychoanalytic theory. Within the class are many sub-classes of treatment, each more or less distinguishable from the others by its techniques, scope, goals, and hypothetical substructure. And within any sub-class the therapist himself is an important variable. Certain characteristics of the patient, the therapist, the setting, and the way these interact, determine what actually happens. The individuality of each sequence of psychotherapy is often seen as its most important feature. The instrument of treatment works differently in different hands and with different patients. Evaluative procedures must take cognisance of this individuality, yet at the same time they must specify as closely as possible the general principles and techniques, so that these can be replicated.

Dynamic psychotherapy is most extensively used for patients suffering from neurotic disabilities. The phenomena of such disorders consist not only of symptoms in the strict sense but also, and oflen more promincently, include problems in personal adjustment, attitudes, and relationships, which are difficult to estimate in a reliable fashion. Moreover, and this is crucial to dynamic psychotherapy, the symptoms and overt evidences of maladjustment can be seen as subordinate to, and deriving from, forces below the surface which can be conceptualized as intrapsychic disturbances. These underlying variables are, by their very nature, inaccessible to direct expression and measurement. So if dynamic psychotherapy is being investigated, serial assessments of the patient are required at three distinct levels. Symptoms and signs must be specified clearly; adequate methods are needed to ascertain attitudes and adjustment to life circumstances, and the circumstances themselves; and there must also be some communicable, reliable, and consistent criteria for describing the psychodynamic configurations which are hypothesized in any particular case, and which the therapist is seeking to change in order to help his patient. To avoid bias and contamination of criteria, data must come from several independent sources. These include the patient himself, a relative or other significant person, the therapist, a general psychiatric examination, and a dynamic assessment. The criteria should be demonstrably sensitive but not capricious, and their interrelationships should be studied. Moreover, they should be such as to permit a clear specification, at the outset, of the objectives of treatment in the individual case. Their application must allow not only for the uniqueness of the patient's problems and their context but also for the possibility of differential improvement and qualitative change in the style of the neurotic disorder. For after any treatment, or with the passage of time, symptoms may improve while life adjustment becomes more restricted, or certain qualities of life adjustment may improve while symptoms remain. Improvement in either or both may seem, to the dynamic psychiatrist, sometimes to be firmly grounded and sometimes to be a false solution associated with persistence of hypothetical underlying conflicts. If the system makes sense, it can be predicted that these will manifest themselves sooner or later in different guise.

How far may these exacting requirements be met in practice? The controlled therapeutic 
trial is the standard instrument for cvaluating a treatment procedure. In its application to dynamic psychotherapy, a series of patients, all demonstrably suitable for the treatment according to explicit criteria, could be randomly allocated to treatment and control groups and assessed comprehensively at various stages before, during, and after treatment. Patients in one control group could have the same amount of a doctor's attention as those in the treatment group, while other control patients could have standard treatment as available in the National Health Service. Such a trial would call for close collaboration between many people with diverse skills. The methodological problems should be settled at the outset, and enormous (but not insuperable) practical difficulties could be anticipated. The Medical Research Council is currently supporting a small-scale exploration into the feasibility of this sort of inquiry. The investigators, from the Maudsley Hospital and the Tavistock Clinic, will shortly present their report, which may be expected to give perspective to some of the central issues.

But the controlled therapeutic trial is not the only, or necessarily the best, way of examining the case for dynamic psychotherapy. Programmes of empirical research are being implemented on an impressive scale in the United States; and some of their findings have highlighted themes which deserve exploration in the very different conditions prevailing in Britain. Close and detailed studies of single cases before, during, and after treatment may yield fruitful hypotheses about the processes and results of treatment, and set the stagc for investigating larger series of patients. The development of indices for describing the salient variables in patient, therapist, and treat- ment is the special provinec of such studies. Applications of the methods of epidemiology and social psychology could throw light on the place occupied by dynamic psychotherapy in the contemporary medical services. It would be valuable, for example, to know something about the various barriers and screening procedures through which patients must pass before being sclected for psychotherapy; about the apparent success or failure of other treatments prescribed before and after psychotherapy; about the extent to which various types of patients persist in psychotherapy once they have started; and about the outcome of neurotic disabilities in patients treated by routine measures.

Philosophical debate about the scientific status of psychoanalysis can do nothing to promote or dismiss the case for its relevance to medicine. What is needed is a concerted attempt to clarify the problems of psychotherapy in practice by high-quality collaborative research which is truly relevant to its subject-matter. Many psychotherapists still tend to invoke authority and to give their language private meanings embodying indistinct assumptions about issues of profound and signal importance. They do less than justice to these matters, and thereby provide nourishment for those who find destructive criticism an absorbing activity. From whatever standpoint the situation is regarded, all should welcome reasoned and impartial inquiry into the efficacy of psychotherapy. Such inquiry is bound to be elaborate and expensive. The sad thing is that the impediments to progress may be attributed not so much to lack of funds as to the common tendency to pre-judge the issue.

ROBERT CAWLEY 